\title{
Archéopages
}

Archéopages

Archéologie et société

36 | 01/2013

Exotismes

\section{Vestiges de bornage antique en Île-de-France et en Picardie. La question des dépôts de vases dans les fossés}

Véronique Pissot, Jean Bruant, Olivier Cotté, Gilles Desrayaud, Sylvain Gautier, Bénédicte Hénon et Louis Hugonnier

\section{(2) OpenEdition}

Journals

Édition électronique

URL : https://journals.openedition.org/archeopages/219

DOI : 10.4000/archeopages. 219

ISSN : 2269-9872

Éditeur

INRAP - Institut national de recherches archéologiques préventives

Édition imprimée

Date de publication : 1 novembre 2013

Pagination : $56-69$

ISSN : 1622-8545

\section{Référence électronique}

Véronique Pissot, Jean Bruant, Olivier Cotté, Gilles Desrayaud, Sylvain Gautier, Bénédicte Hénon et Louis Hugonnier, "Vestiges de bornage antique en Île-de-France et en Picardie. La question des dépôts de vases dans les fossés », Archéopages [En ligne], 36 | 01/2013, mis en ligne le 01 janvier 2015, consulté le 22 janvier 2022. URL : http://journals.openedition.org/archeopages/219 ; DOI : https://doi.org/10.4000/archeopages.219 


\title{
Vestiges de bornage antique en Île-de-France et en Picardie La question des dépôts de vases dans les fossés
}

\author{
Véronique Pissot Inrap \\ Jean Bruant Inrap, Uмr 7041 «Archéologies et sciences de l'Antiquité» \\ Olivier Cotté Inrap, UMR 7324 « Cités, territoires, environnement et sociétés » \\ Gilles Desrayaud Inrap \\ Sylvain Gautier Inrap \\ Bénédicte Hénon Inrap, UMR 8215 « Trajectoires de la sédentarisation à l'État (VII-1-1er millénaire av. J.-C.) » \\ Louis Hugonnier Inrap
}

Une quarantaine de sites en Île-de-France et en Picardie a révélé la présence de vases entiers, parfois associés à des pierres, tuiles ou restes de faune, au niveau d'apparition de fossés. À première vue, ces dépôts pourraient être interprétés comme de simples rejets domestiques. Ils évoquent cependant une pratique relative au bornage, décrite par les arpenteurs antiques et toujours vivante. La délimitation des parcelles consiste non seulement à fixer des repères en surface, mais éventuellement à placer sous ces derniers un témoin en matériau non périssable qui servira de preuve en cas de litige entre propriétaires. L'étude de ces marqueurs de limites, caractérisés par une extrême diversité comme en témoignent les traités d'arpentage (Favory, Gonzales, Guillaumin, Robin, 1995, p. 279-280), ne saurait se réduire aux seuls dépôts de vases en terre cuite. Toutefois, leur analyse constitue un bon point de départ pour aborder un domaine encore peu exploité en archéologie préventive.

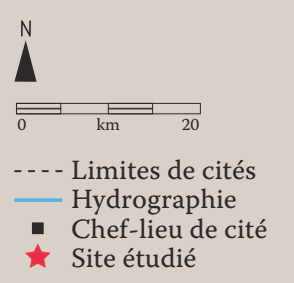

1. Localisation des sites étudiés.

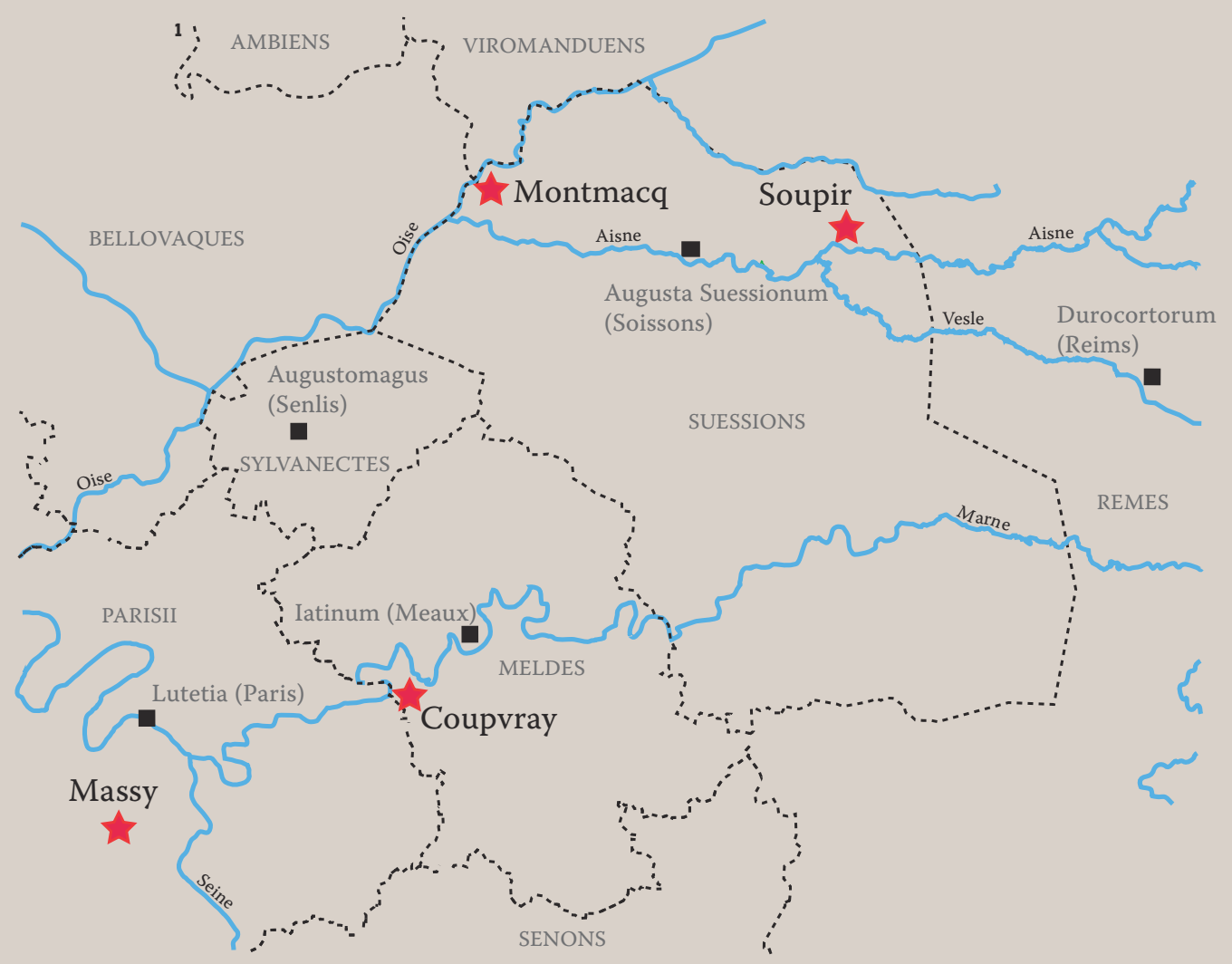




\section{État des lieux}

La recherche sur l'utilisation de récipients céramiques à des fins de bornage a été initiée en France par la publication du site de Sept-Fonts dans l'Hérault (Mauné, 1992, 1997). Cet établissement rural a fait l'objet d'une découverte remarquable : deux alignements perpendiculaires de cols d'amphores retournés surmontant une fosse à offrandes, scellée par une couche de pierres soigneusement tassées. Cet agencement particulier a conduit l'auteur à un rapprochement avec un texte de l'arpenteur Siculus Flaccus (II ${ }^{\mathrm{e}}$ siècle). L'emploi de céramiques au sein de fossés parcellaires a été mis en évidence ailleurs en Narbonnaise et également en Tarraconaise (Vidal, Petitot, 2003 ; Gurrera, Gallemí, 1994 ; Olesti, Massó, 1997).

En Île-de-France, la découverte, dans un parcellaire antique, d'un fossé ayant livré une série de récipients complets a été l'occasion d'un rapprochement entre techniques d'arpentage et données de terrain (Robert, 1996). La question ne sera reprise qu'en 2011, dans le cadre d'une présentation de trois sites franciliens présentant ce même type de dépôts au sein de fossés à fonction limitative (Bruant et al., 2011). L'objectif de cette contribution était d'attirer l'attention sur un phénomène encore peu pris en compte afin que de nouvelles occurrences puissent être signalées et comparées aux exemples déjà connus; dans le cadre de la Société française d'étude de la céramique antique en Gaule, l'attention s'était portée en priorité sur les récipients sans que ne soient véritablement développés les aspects contextuels, même s'il paraît évident que l'objet céramique ne peut être dissocié de la structure dans laquelle il est enfoui. Ces lacunes relatives à l'environnement archéologique des « dépôts » n'étaient pas seulement dues au contexte particulier d'une publication dans une revue de céramologie, mais aussi et surtout à une insuffisance de la collecte des informations contextuelles dès la phase de terrain. La découverte de céramiques dans un fossé n'évoque pas a priori autre chose qu'un simple rejet domestique, surtout si, de surcroît, aucune grille de lecture n'a été élaborée pour guider les gestes techniques sur le terrain.

Plus généralement, les conditions de réalisation des diagnostics, aussi bien que les objectifs affichés, permettent rarement de mettre en œuvre les moyens susceptibles d'accéder à un niveau d'analyse satisfaisant, lequel doit dépasser le cadre de la seule emprise explorée. De même, les délais de postfouille sont insuffisants pour engager des études micromorphologiques ainsi qu'une analyse fine des dynamiques de comblement des fossés.

La rareté des publications sur le sujet donne tout son sens à cette deuxième contribution consacrée à l'analyse de quatre sites, picards et franciliens [ill. 1] qui, toutefois, n'offrent pas tous la même valeur informative en raison de la prise en compte ou non de la problématique dès la phase de diagnostic : cette variabilité dans la collecte et le traitement de l'information peut d'ailleurs éventuellement en biaiser l'interprétation. Dans tous les cas, ces nouveaux exemples conduisent à s'interroger sur la manière de prendre en compte ces vestiges discrets, dont le potentiel informatif dépasse largement les questions purement céramologiques et chronologiques.

\section{Soupir / « Petite Forêt » (Aisne)}

Depuis les années 1980, la plaine de Soupir/ Moussy-Verneuil (Aisne) a fait l'objet de recherches archéologiques qui ont permis une reconnaissance extensive du territoire. Pour la période romaine, trois sites d'importance inégale ont été reconnus. La villa de Soupir est installée sur une petite éminence graveleuse [ill. 2]. Sur les 3 ha d'occupation, on observe un déplacement progressif de l'habitat du nord-est vers le sud-est. À $1,70 \mathrm{~km}$ vers l'est, une petite installation du HautEmpire, dont la fonction n'est pas déterminée, se développe sur environ 0,40 ha. À $500 \mathrm{~m}$ au sud-est, une grande villa, qui s'étend sur plus de 6 ha, vient d'être découverte (Robert, rapport en cours). Un important réseau de fossés parcellaires est mis en place entre ces établissements, en lien avec les différentes activités humaines et/ou le drainage, en particulier sur les sols marqués par un substrat plus argileux. À $300 \mathrm{~m}$ à l'est de l'habitat de Soupir, deux fossés, datés du Haut-Empire et distants de $120 \mathrm{~m}$, ont livré dans leur comblement supérieur des dépôts céramiques [ill. 3]. Ces deux fossés ont été fouillés mécaniquement mais intégralement, ce qui a permis une localisation précise des dépôts (Hénon et al., 2001).

Le fossé 18 , large de $1,50 \mathrm{~m}$ pour $0,50 \mathrm{~m}$ de profondeur, a fait l'objet de plusieurs creusements successifs. Les dépôts de céramiques débutent à $13 \mathrm{~m}$ de la jonction avec un fossé orienté est-ouest et se développent sur $60 \mathrm{~m}$ de long environ. On observe une certaine régularité dans la disposition de ces vases distants de 4 à $8 \mathrm{~m}$, souvent $6 \mathrm{~m}$ [ill. 3 et 13].

Sept récipients presque complets y ont été déposés. La vaisselle fine régionale est majoritaire : trois pots biconiques $\left(\mathrm{n}^{\text {os }} 18.1,18.3\right.$ et 18.5) et trois pots à col concave et panse globulaire ou ovoïde $\left(\mathrm{n}^{\mathrm{os}} 18.4,18.6\right.$ et 18.7$)$. Le dernier vase est un pot à cuire ayant conservé des traces d'utilisation (éclats de surface, plages noircies). En outre, le fossé a livré les fragments d'une assiette de type Dragendorff 18/31 en sigillée d'Argonne ( $\mathrm{n}^{\circ}$ 18.9), des fonds et bords de récipients variés (vaisselle fine, culinaire et de conservation - dolium $\mathrm{n}^{\circ}$ 18.14). L'ensemble est attribuable à la première moitié du II ${ }^{e}$ siècle, la vaisselle sigillée incitant à privilégier une datation basse, autour des années 120-150.

Au bord du fossé 18 , au niveau de sa jonction avec un fossé perpendiculaire orienté est-ouest, une incinération, datable également de la première moitié $\mathrm{du} \mathrm{II}^{\mathrm{e}}$ siècle, a livré, outre les ossements du 


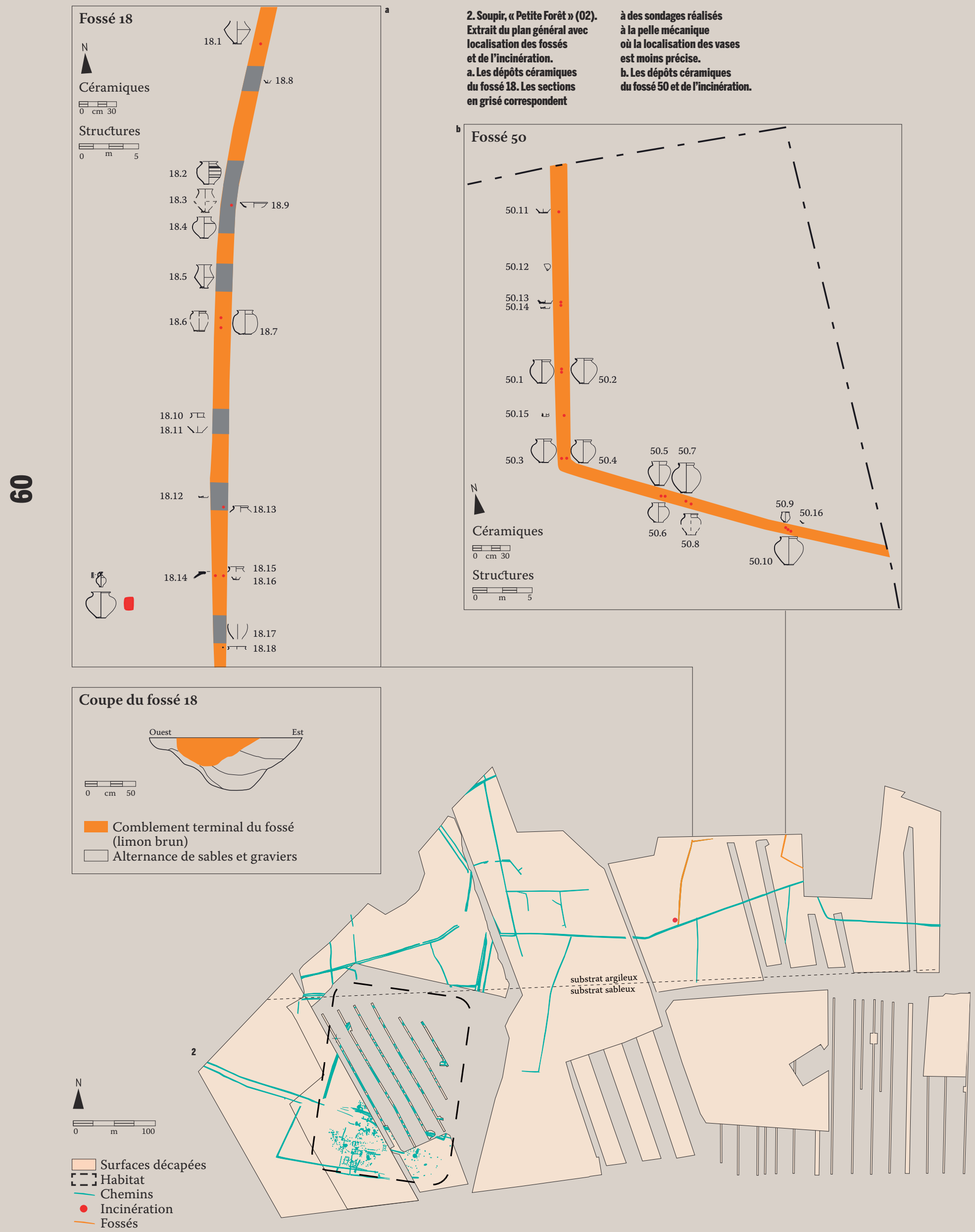


défunt, des restes de caprinés, deux vases calcinés (un pot à cuire et une cruche de faible module) et une monnaie d'Hadrien ou Antonin le Pieux (soit des années 117 à 161).

Le fossé 50 se situe à une centaine de mètres à l'est du précédent. Il forme un angle (enclos ?) dont deux tronçons incomplets ont été décapés, soit $55 \mathrm{~m}$ linéaires. Ce fossé large de $1 \mathrm{~m}$ atteint une profondeur de o,40 m. Deux phases de comblements successifs se distinguent. Seize vases ont été déposés dans ce fossé, par paires dans au moins quatre cas. Deux d'entre eux sont déposés à l'angle, puis les dépôts suivants sont espacés de 6 à 8 m environ [ill. 3 et 13].

Les vases les mieux conservés sont au nombre de dix. Leur composition fonctionnelle est tout à fait différente de celle du fossé 18. Dans les deux cas, il s'agit de formes fermées exclusivement, mais la vaisselle culinaire est ici majoritaire. On dénombre six pots à cuire, en tous points semblables ( $\left.\mathrm{n}^{\text {os }} 50.1,50.2,50.3,50.4,50.7,50.10\right)$. À une exception près ( $\left.\mathrm{n}^{\circ} 50.7\right)$, ils présentent des traces d'utilisation (suie). Les quatre derniers vases correspondent à des productions fines régionales : trois gobelets ( ${ }^{\text {os }} 50.5,50.6$ et 50.9), un pot $\left(n^{\circ} 50.8\right)$. Il est notable que trois de ces récipients $\left(\mathrm{n}^{\text {os }} 50.6,50.8\right.$ et 50.9) présentent les stigmates d'un passage au feu alors même qu'ils ne sont pas destinés à un usage culinaire.

D'autres fragments ont été trouvés : des fonds de récipients variés, de production régionale (pot à cuire, bouteille, amphore) ou importée (sigillée estampillée - VS.F, $\mathrm{n}^{\circ}$ 50.12).

L'ensemble de ce mobilier est datable de la seconde moitié du ${ }_{11}^{\mathrm{e}}$ siècle (des années 150-170 ?). Il semble donc que, dans chacun des fossés, l'enfouissement des vases se soit effectué sur un court laps de temps, sinon en une seule fois, au cours de la première moitié $\mathrm{du} \mathrm{II}^{\mathrm{e}}$ siècle pour le premier, de la deuxième moitié pour le second.

On peut se demander si ces dépôts (et la sépulture?) ne répondaient pas à une volonté de pérenniser des limites alors que les fossés, presque comblés, n'étaient plus ou peu visibles et que leur entretien en tant que fossés n'était plus nécessaire. Il pourrait alors s'agir de limites bornées entre deux domaines proches et potentiellement mitoyens, ceux de Soupir et Moussy, qui semblent occupés à la même période et se partagent un même terroir à $2 \mathrm{~km}$ de distance.

\section{Massy / « Rue du Pérou » (Essonne)}

À Massy, un tronçon de la voie antique Cenabum-Lutetia (Orléans-Paris) a été abordé lors d'une fouille préventive au lieu-dit « Rue du Pérou » (Bruant et al., 2008 ; Bruant, 2009 ; Bruant et al., 2011) [ill. 4]. Les travaux ont documenté les premières étapes du modelé de la voie et des systèmes drainants associés qui évoluent à partir d'un chemin creux attribuable à La Tène finale jusqu'à la mise en place, à la période antique et après plusieurs phases intermédiaires, d'une véritable voie empierrée bordée de fossés perdurant jusqu'à l'époque moderne. La dernière phase observée durant la période romaine est marquée par une évolution des fossés-limites de la voie assurant son drainage, voire par la matérialisation de son emprise publique (Coulon, 2007, p. 86-89) vers des structures drainantes apparentées aux noues qui bordent certaines voiries actuelles.

Malgré un éloignement relatif des occupations antiques (300 m environ), du mobilier archéologique a été mis en évidence dans le comblement des noues [ill. 5 et 6]. Il s'agit de dépôts associant moellons de pierre, céramiques parfois entières et concentrations plus ou moins denses de restes fauniques. L'observation de ce phénomène dès la phase de diagnostic a permis une approche spatiale et stratigraphique mettant en rapport les éléments mis au jour avec l'évolution du modelé de la voie et de ses aménagements.

La présence de moellons de pierre (178) a été observée presque en continu en partie sommitale du comblement des fossés bordant la voie. Ces éléments sont majoritairement représentés par des moellons bruts de meulière, déposés isolément ou en chapelets plus ou moins distendus.

Près de 500 restes de faune proviennent de squelettes plus ou moins complets parmi lesquels on compte au minimum quatre bœufs (Bos taurus) et quatre chevaux (Equus caballus) dont les carcasses n'ont pas été exploitées [ill. 7]. Leurs ossements ont été découverts dispersés le long des noues, individuellement ou en morceaux de carcasses. Le phénomène s'observe plus particulièrement pour les boufs, avec une dispersion pouvant s'étendre jusqu'à $75 \mathrm{~m}$ de long pour le même animal. Les carcasses semblent avoir été disloquées avant enfouissement comme l'indique l'état de conservation du périoste. Deux scénarios sont possibles : les cadavres ont été rejetés dans les fossés avant que leurs restes ne soient dispersés, mais ils peuvent également avoir été déposés ailleurs, puis, dans un second temps, rejetés par morceaux de carcasses le long de la voie, participant ainsi à son bornage.

Les dépôts de céramiques se composent d'au moins 20 céramiques découvertes, à deux exceptions près, dans le fossé-limite occidental [ill. 6 et 13]. Quatre vases sont presque complets ( $n^{\text {os }} 1$ à 4). L'un d'eux a vu sa lèvre découpée à la base du $\mathrm{col}\left(\mathrm{n}^{\circ} 4\right)$. Cette première série de vases provient d'un tronçon du fossé où les moellons de pierre sont les plus rares. Une deuxième série de céramiques présente un moins bon état de conservation : seule nous est parvenue la partie supérieure ou inférieure du récipient, sans trace de découpe manifeste ( $\mathrm{n}^{\text {os }} 5$ à 13$)$. Le reste du mobilier se compose de petits fragments, notamment de deux bords et d'un fond ( $\mathrm{n}^{\text {os }} 14$ à 16$)$.

La vaisselle culinaire est majoritaire (14 vases). Il s'agit de formes fermées pour l'essentiel, destinées à aller au feu. Ces pots à cuire montrent 
Phase 5 :

Période augustéenne $-\mathbf{1}^{\mathrm{re}}$ moitié $\mathrm{I}^{\mathrm{er}} \mathrm{S}$.

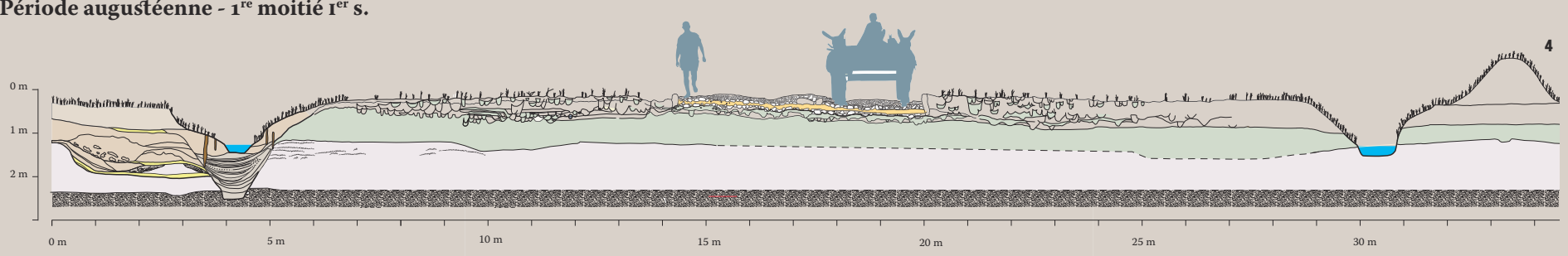

Aménagement d'une voirie empierrée dotée de deux fossés bordiers et d'accotements également utilisés pour la circulation.

Phase 6:

$2^{\text {nde }}$ moitié I $^{\text {er }}$ S. - III ${ }^{\mathrm{e}}$ S.

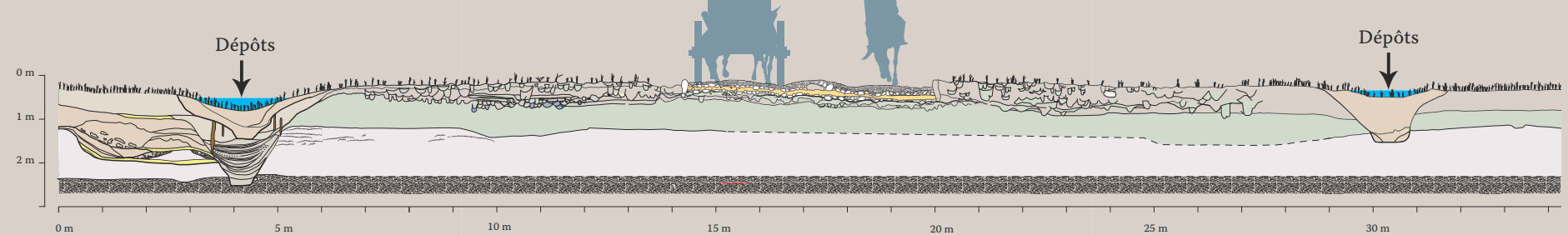

Comblement massif des fossés bordiers.Transformation en noues.

Dépôts en chapelet de céramiques parfois entières, d'ossements de faune et de moellons de meulière.

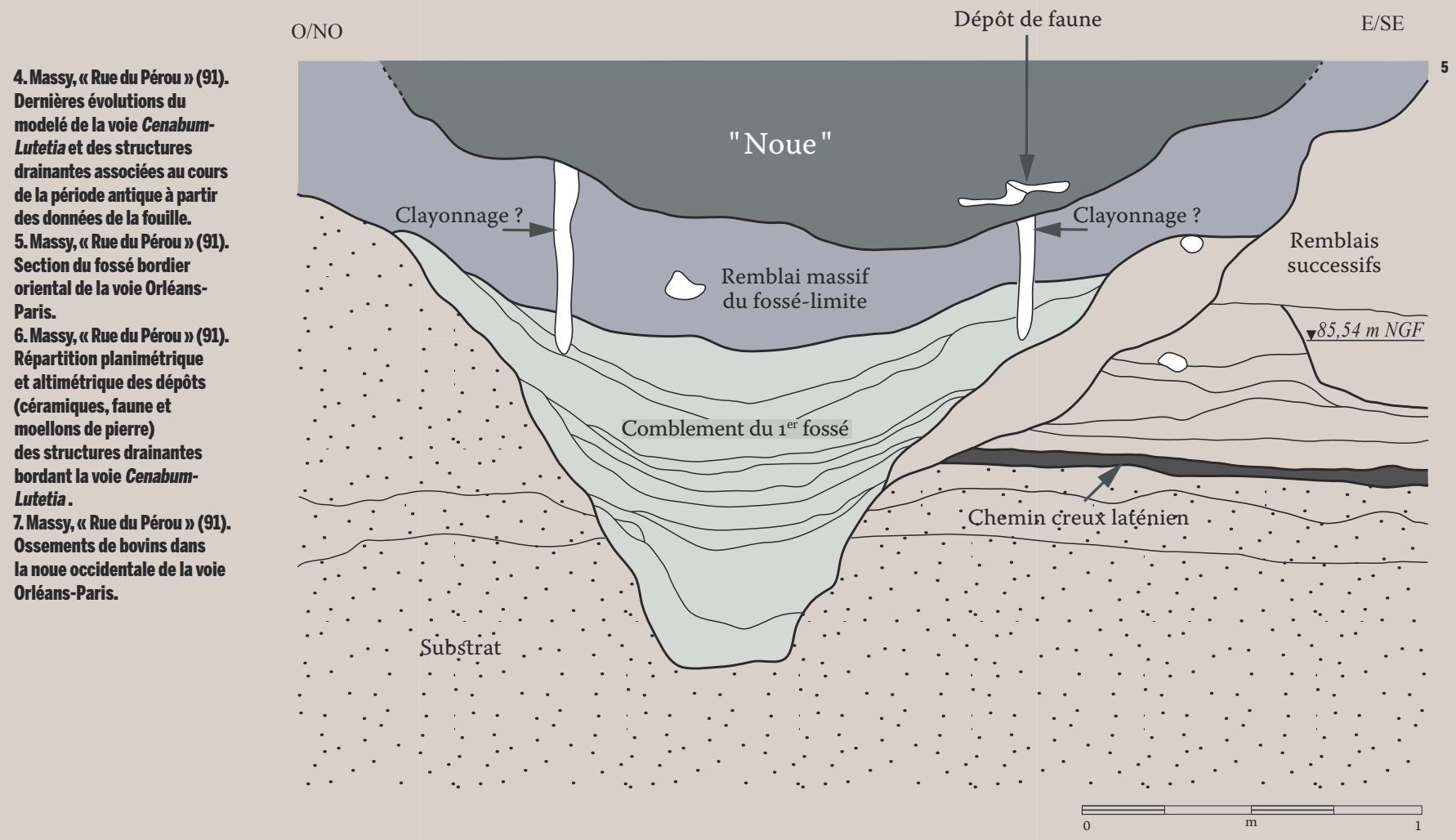



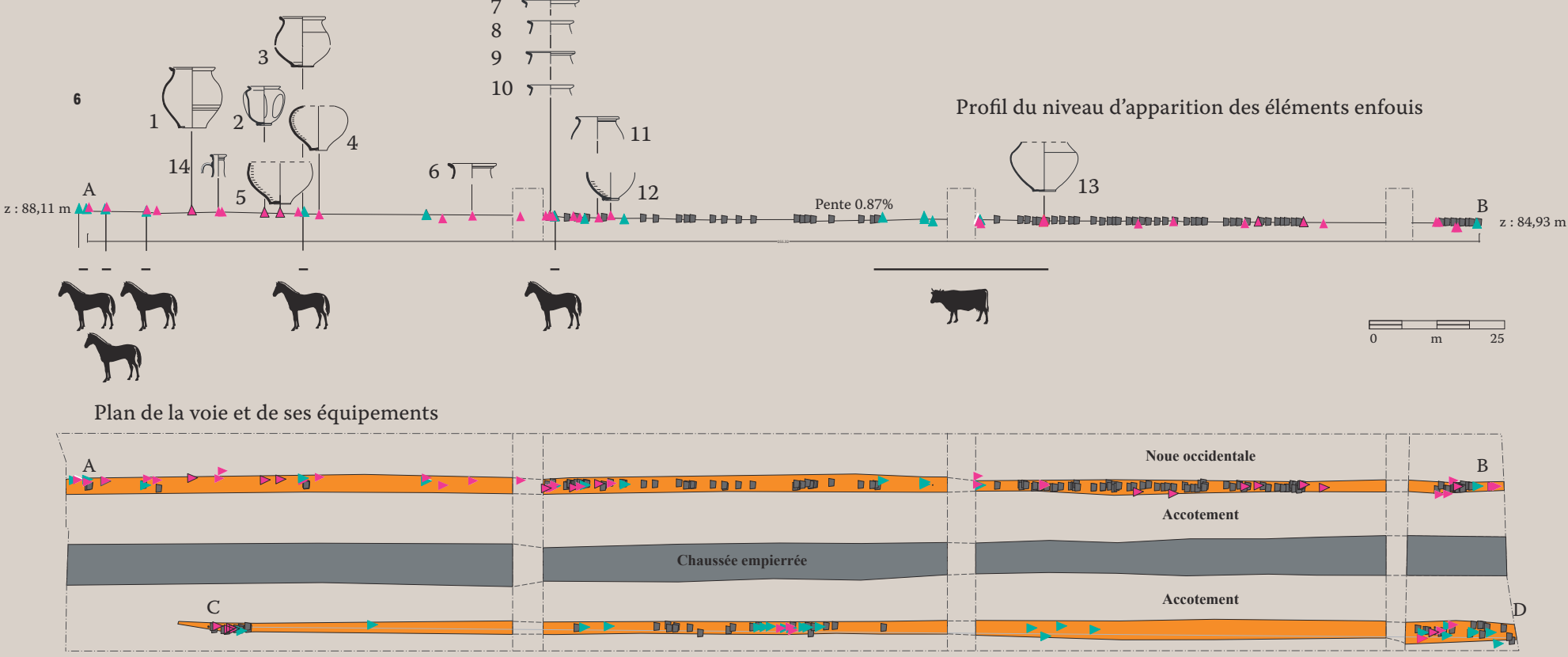

SSO
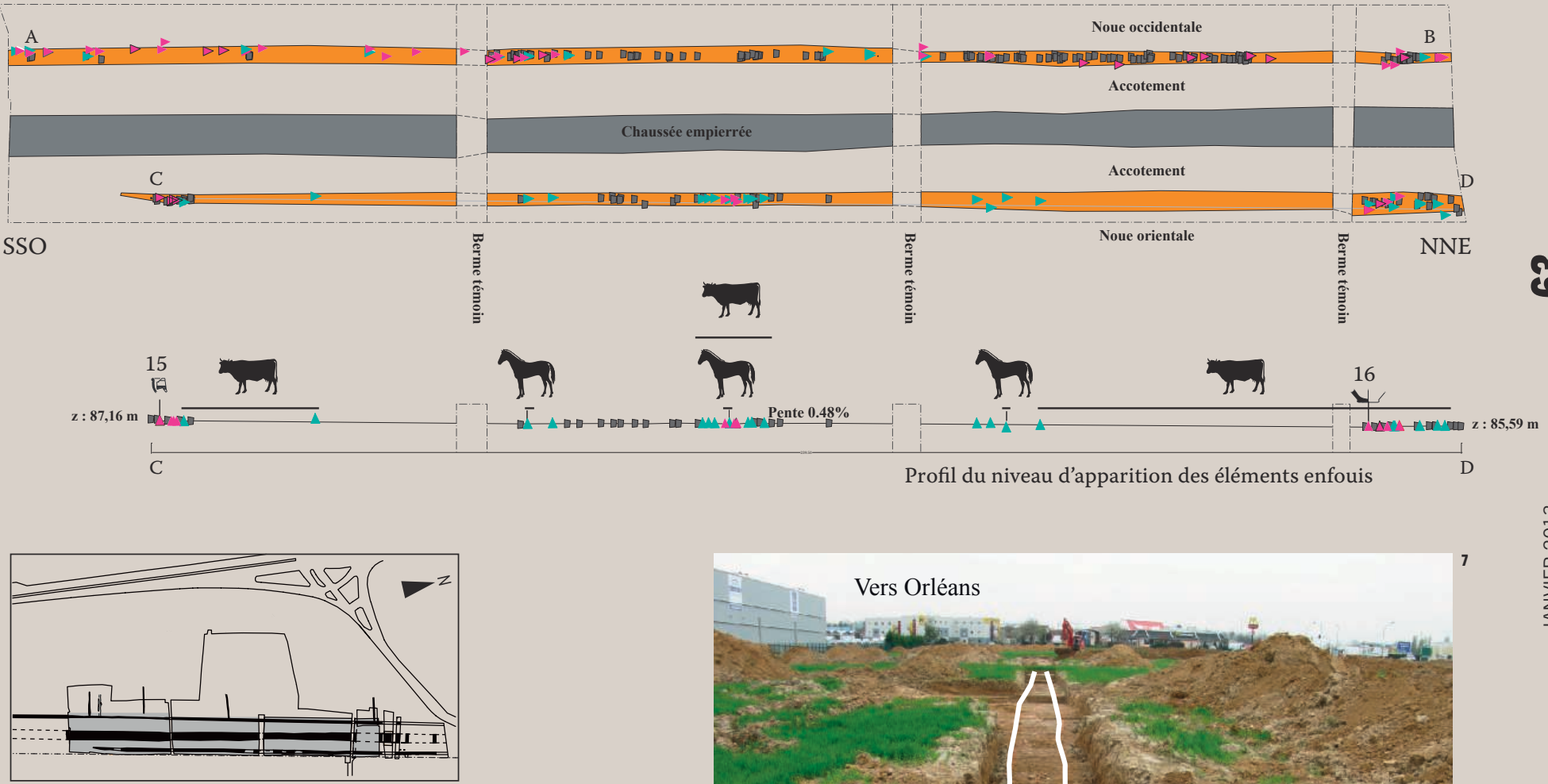

Fossés-limites

Chaussée empierrée

$\Delta$ Faune

A Céramique gallo-romaine

- Moellons de pierre

Ossements de bouf (Bos taurus)

T) Ossements de cheval (Equus caballus)
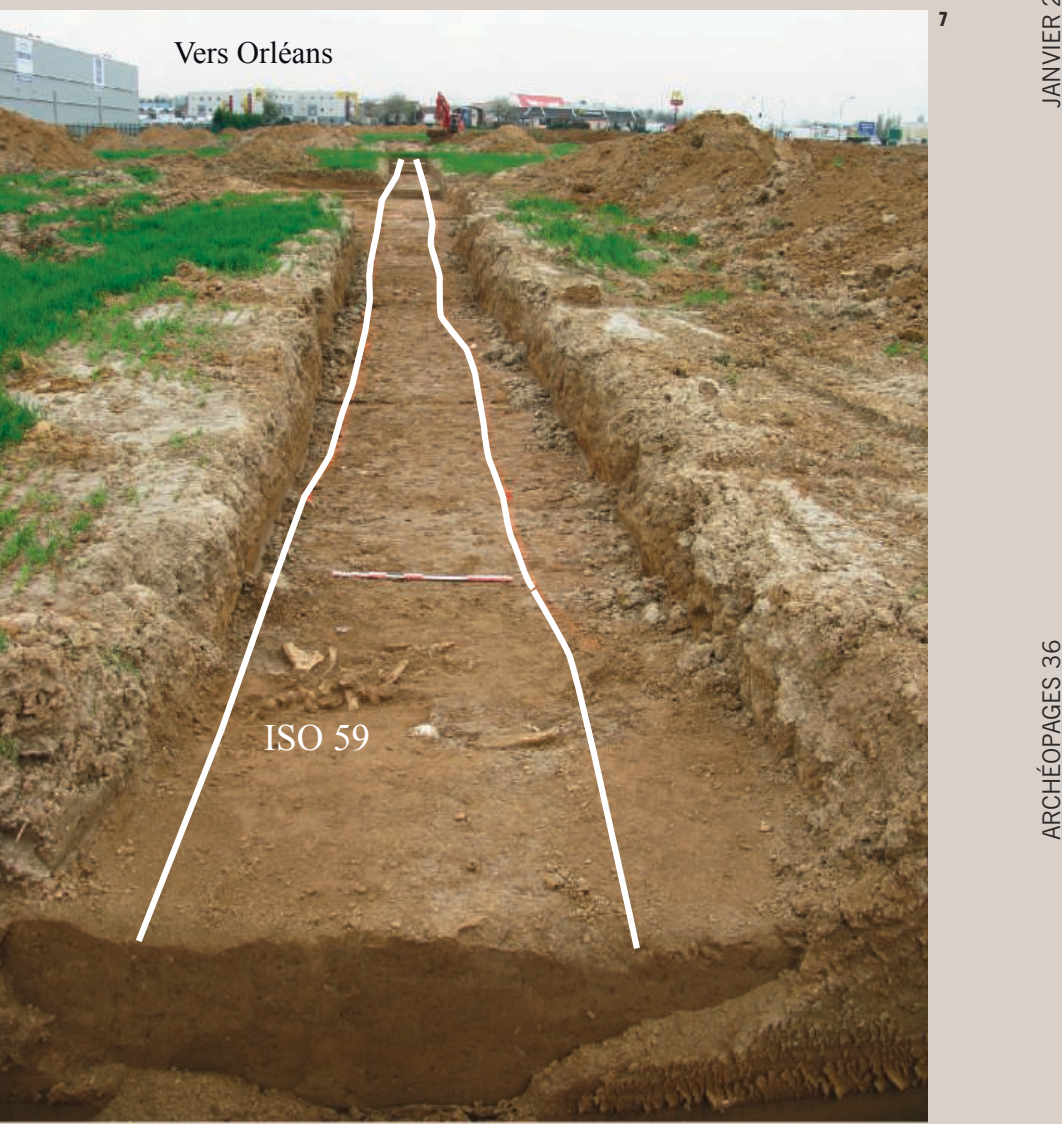

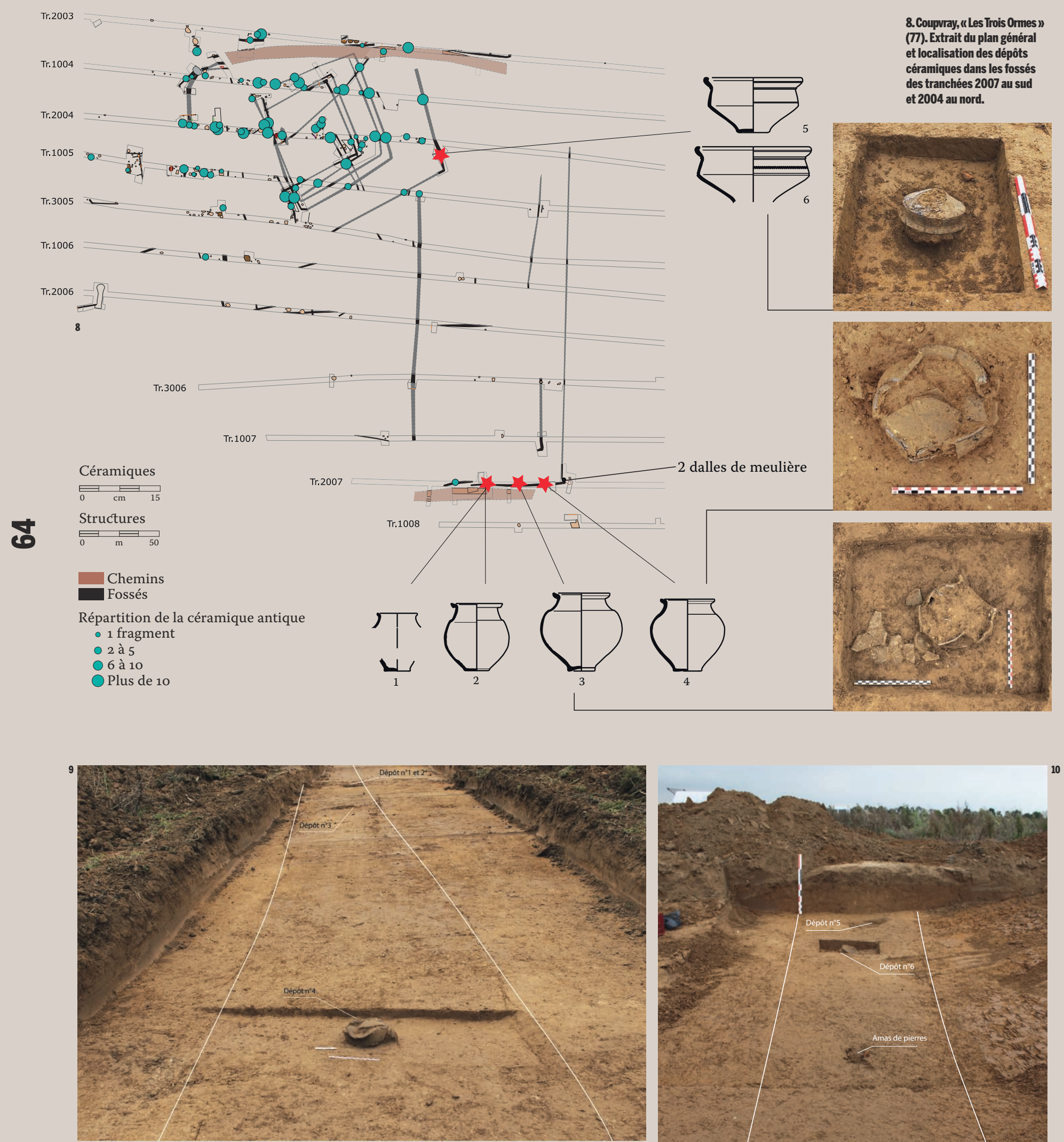

9. Coupvray, «L Les Trois

Ormes ॥ (77). Alignement

des dépôts du fossé 2007.3,

vu depuis l'est.

10. Coupvray, « Les Trois

Ormes ॥ (77). Alignement

des dépôts du fossé 2004.9,

vu depuis le nord.

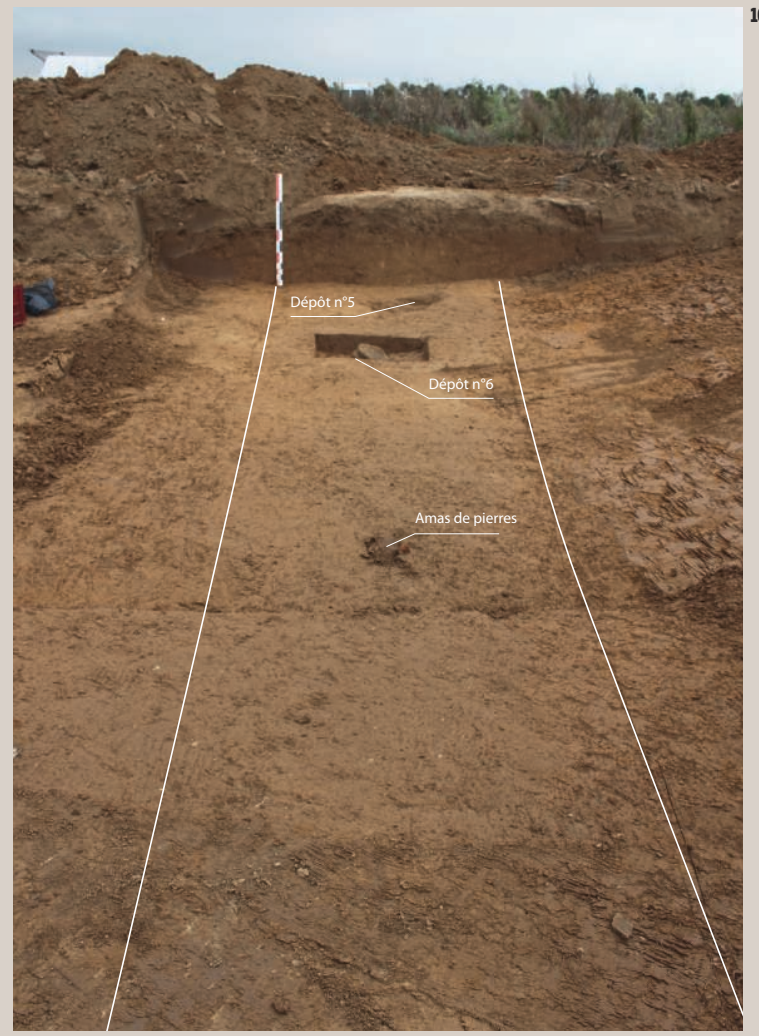


tous des traces d'utilisation (suie, caramels alimentaires). Les six derniers vases sont liés au service : une coupe en sigillée de Gaule centrale $\left(n^{\circ} 15\right)$, un gobelet $\left(n^{\circ} 2\right)$, une bouteille $\left(n^{\circ} 4\right)$ et trois cruches ( $\mathrm{n}^{\text {os }} 12,14$ et 16$)$. À quatre exceptions près, les vases sont de couleur sombre. On retiendra enfin que l'ensemble est formé presque exclusivement de vaisselle domestique de production locale.

Les céramiques les mieux conservées donnent un éventail de datations assez large, avec des intervalles couvrant la seconde moitié du ${ }_{\mathrm{i}}^{\mathrm{er}}$ siècle et la première moitié $d u_{\text {II }}{ }^{\mathrm{e}}$ siècle $\left(\mathrm{n}^{\mathrm{os}} 4,6,12\right.$ et 14$)$, le ${ }_{\text {II }}^{\mathrm{e}}$ siècle $\left(\mathrm{n}^{\mathrm{os}} 1,2\right.$ et 3$)$ ainsi que la seconde moitié $\mathrm{du}_{{ }_{\mathrm{II}}}^{\mathrm{e}}$ et le ${ }_{\mathrm{III}}^{\mathrm{e}}$ siècle $\left(\mathrm{n}^{\circ} 5, \mathrm{n}^{\mathrm{os}} 7\right.$ à $11, \mathrm{n}^{\text {os }} 13,15$ et 16$)$, ce qui évoque a priori des enfouissements successifs plutôt qu'une unique phase de dépôt.

Dans un contexte de remblai partiel et de colmatage progressif des fossés drainants marqué par l'atténuation ou la disparition brutale du ou des merlons bordant les fossés, la nécessité de réaffirmer les limites de la voie a pu motiver la mise en place de repères enfouis, utilisés pour pérenniser l'emplacement de bornes visibles en surface. Différentes phases de marquage pourraient expliquer le probable étalement dans le temps des dépôts de céramiques.

Il est également possible d'envisager que ces éléments enfouis aient servi de repères pour l'entretien des structures drainantes. Les dépôts ont pu aider à matérialiser une cote à ne pas dépasser, afin de maintenir la faible déclivité calculée pour infiltrer la plus grande quantité d'eau sur place et, ainsi, éviter que cette dernière n'afflue massivement vers l'aval. On a pu également vouloir empêcher que le surcreusement du fond n'entraîne un élargissement des bords et une diminution de la largeur des accotements de la chaussée.

Ces hypothèses pourraient s'accommoder de l'amplitude chronologique du corpus des céramiques déposées puisque, durant ce laps de temps, plusieurs campagnes d'entretien (curage, faucardage, voire brûlage des végétaux) ont pu avoir lieu. Les céramiques déposées à cette occasion (bas de gamme, à formes hautes, ayant vraisemblablement contenu les aliments les plus couramment consommés de type bouillie, mais aussi des liquides) pourraient ainsi renvoyer à un recrutement social particulier, auquel le personnel chargé des travaux semble le mieux correspondre.

\section{Coupvray / « Les Trois Ormes » (Seine-et- Marne)}

Coupvray a fait l'objet d'un diagnostic sur une surface de plus de 60 ha (Durand, 2012). Dans la zone sud-ouest de l'emprise, une occupation antique a été mise en évidence [ill. 8] succédant à une occupation de La Tène finale marquée par un, voire deux enclos quadrangulaires et des puits d'extraction de minerai de fer. La période romaine s'illustre par la construction de trois ou quatre nouveaux enclos, d'un probable bâtiment en leur centre et de structures annexes. Occupé jusqu'au ${ }_{\text {III }}{ }^{\mathrm{e}}$ siècle, le site ne semble réinvesti qu'à partir du haut Moyen Âge (vII ${ }^{\mathrm{e}}$-xi $\mathrm{e}^{\mathrm{e}}$ siècles).

L'examen de la répartition spatiale de la céramique antique a révélé une forte concentration de mobilier dans le secteur des enclos. Aux marges de cette zone densément occupée, des vases presque complets ont été trouvés dans deux tronçons de fossés [ill. 8 à 10, 13] : l'un au nord-est (tranchée 2004) et l'autre au sud (tranchée 2007).

Le fossé sud est situé à environ $180 \mathrm{~m}$ de l'espace enclos. Apparu à o,40 m de la surface décapée, il est comblé d'un limon argilo-sableux de couleur brun-beige, extrêmement compact, sur une profondeur de $0,24 \mathrm{~m}$. D'une largeur initiale de $0,70 \mathrm{~m}$, il s'élargit vers l'est pour atteindre 1,20 m. Quatre vases ont été découverts lors du décapage, au niveau d'apparition de la structure. Trois vases reposaient couchés sur la panse $\left(\mathrm{n}^{\mathrm{os}} 2,3\right.$ et 4$)$. Il s'agit de formes fermées exclusivement, provenant d'ateliers locaux. Les trois exemplaires les mieux conservés ( $\mathrm{n}^{\text {os }} 2$ à 4 ) présentent le profil classique des pots à cuire de ce secteur géographique au cours des années 80-150 environ. Ils présentent les traces d'un passage au feu lié à leur utilisation. Le vase le moins bien conservé $\left(n^{\circ} 1\right)$, quoique techniquement apparenté aux précédents, est d'un profil différent. Il est difficile de préciser si les deux vases ( $\mathrm{n}^{\text {os }} 1$ et 2 ) ont fait l'objet d'un unique dépôt ou de deux dépôts successifs.

Le second dépôt a été mis en évidence dans un tronçon de fossé du plus grand des enclos délimitant la zone de forte occupation. La structure, d'une largeur de 1,90 m pour $0,60 \mathrm{~m}$ de profondeur, présente un fond plat et un profil évasé. Deux vases situés dans le comblement du fossé, à son niveau d'apparition, y ont été découverts. L'un d'eux reposait à l'envers, sur la lèvre $\left(n^{\circ} 6\right)$. L'exemplaire le mieux conservé présente les stigmates d'un passage au feu au moment de son utilisation : arrachements de pâte en surface et zones fortement noircies. Les deux formes, strictement identiques, correspondent à des jattes carénées, fréquentes dans la région de Meaux à la fin du ${ }_{\text {II }}{ }^{\mathrm{e}}$ siècle et $\mathrm{au}_{\mathrm{III}}^{\mathrm{e}}$ siècle.

Le site de Coupvray offre l'exemple de dépôts datant pour une première série de la fin du $\mathrm{r}^{\mathrm{er}} \mathrm{ou}$ de la première moitié du ${ }_{\text {II }}{ }^{\mathrm{e}}$ siècle et pour l'autre de la fin du II $^{\mathrm{e}}$ ou du III $^{\mathrm{e}}$ siècle.

Le fossé sud, relativement éloigné de la zone d'habitat, est assimilable à un fossé parcellaire au sein duquel l'enfouissement d'objets en matériaux non périssables (céramique, pierres) aura permis de conserver le tracé.

Le fossé oriental a, quant à lui, livré deux vases. En première analyse, ce dépôt peut sembler moins pertinent que le précédent en raison de la proximité de l'habitat, qui ne permet pas d'exclure l'hypothèse d'un simple rejet domestique de vaisselle usagée. Toutefois, ce fossé a pu constituer une limite forte, pour l'habitat antique d'une part, jusqu'au ${ }_{\text {III }}{ }^{\mathrm{e}}$ siècle, date de son abandon, mais aussi, 


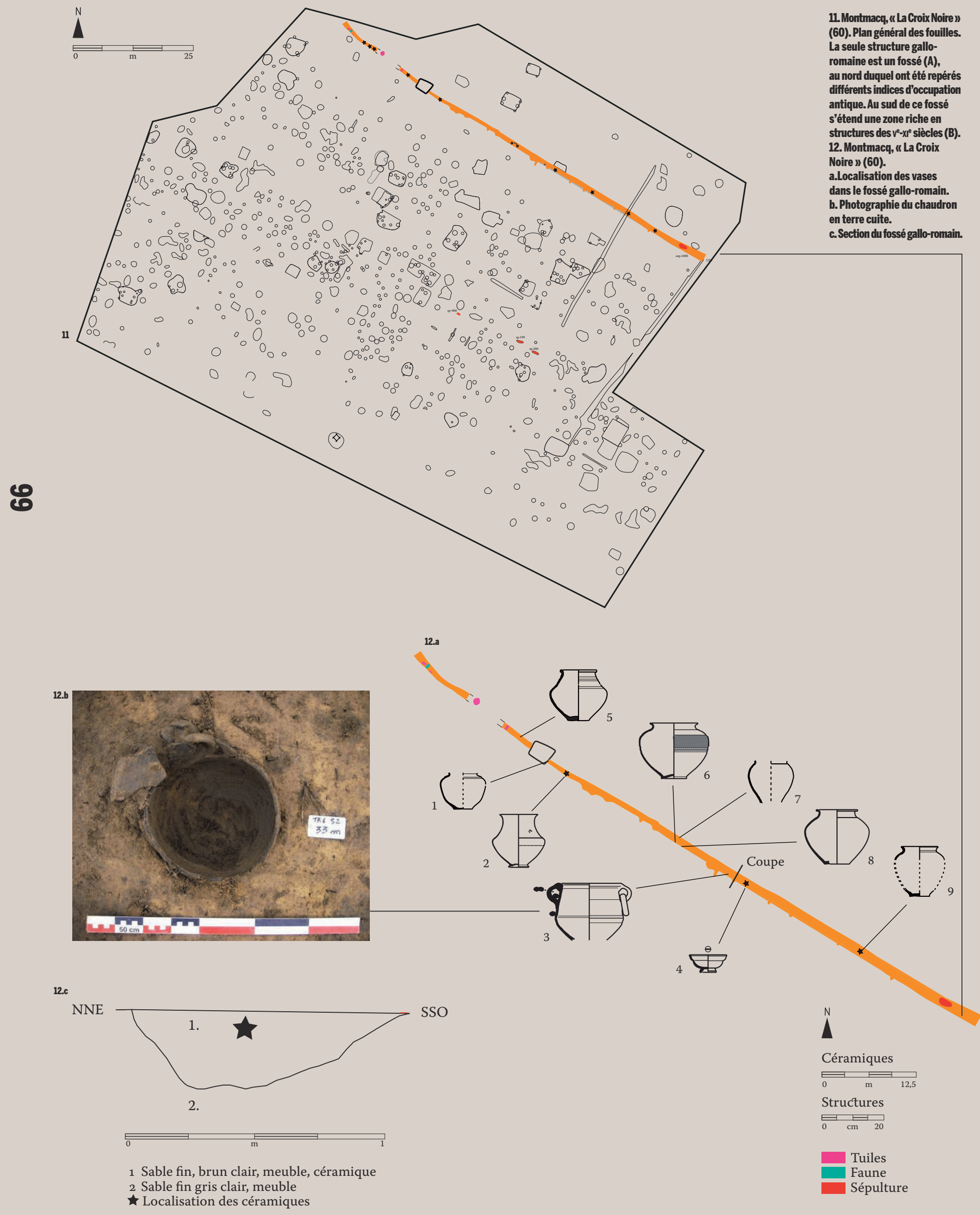


semble-t-il, pour la nécropole postérieure, où 31 sépultures sont concentrées dans ce grand enclos, sans doute encore visible longtemps après son abandon.

\section{Montmacq / « La Croix Noire » (Oise)}

La fouille, qui fait suite à la découverte d'une triple occupation historique (gallo-romaine, mérovingienne et carolingienne tardive) lors du diagnostic (Paris, 2012), a mis au jour près de 900 structures relevant majoritairement de la période médiévale $\left(\mathrm{v}^{\mathrm{e}}-\mathrm{xI}^{\mathrm{e}}\right.$ siècles). Une unique structure gallo-romaine, un fossé, a été identifiée [ill. 11].

Le fossé antique à profil évasé a été suivi sur $87 \mathrm{~m}$. Il est large de 0,40 à $0,70 \mathrm{~m}$ et présente une profondeur moyenne de 0,70 à $0,80 \mathrm{~m}$. Dans sa partie sommitale (les 20 premiers centimètres) sont apparus des marqueurs de nature différente [ill. 12 et 13].

Un ensemble d'au moins huit ou neuf vases en place, quatre ayant été trouvés au cours du diagnostic et étudiés par Cyril Chaidron $\left(\mathrm{n}^{\mathrm{os}} 3,6,7\right.$ et 8), provient du sommet du fossé. Trois pots en terra nigra régionale sont illustrés : un exemplaire est décoré ( $\mathrm{n}^{\circ} 6$; type Deru P. 28 var. ; Deru, 1997), un autre porte une inscription gravée après cuisson (lecture XI ou IX; $\mathrm{n}^{\circ} 2$; type Deru P. 55) et le dernier présente une face interne corrodée $\left(\mathrm{n}^{\circ} 9\right.$; type Deru P. 27). L'ensemble compte aussi quatre pots à usage culinaire portant des traces d'utilisation : trois pots en pâte grossière $\left(\mathrm{n}^{\mathrm{os}} 1,7 \mathrm{et}\right.$ 8 , ce dernier n'étant que très partiellement conservé) et un dernier exemplaire, dont la hauteur n'est que restituée, réalisé dans une pâte granuleuse $\left(n^{\circ} 5\right)$. Les deux derniers vases tranchent avec cette série de formes fermées de couleur sombre : une coupelle en sigillée sudgauloise ( $\mathrm{n}^{\circ} 4$; Drag. 27 ; estampille du potier Dontio) et un grand récipient imitant la forme des chaudrons métalliques $\left(\mathrm{n}^{\circ} 3\right)$. Ce dernier, une forme rare en Picardie, est typique des contextes funéraires du nord-est de la Gaule Belgique. Néanmoins, ses caractéristiques techniques (une pâte grise limoneuse) trahissent une origine locale.

Aucun vase n'est tout à fait complet après remontage, et le cas du chaudron prouve qu'au moins un vase aura été enfoui dans un état fragmentaire : il lui manque le fond, alors même qu'il a été déposé dans son sens habituel d'utilisation (ouverture vers le haut).

On situe l'activité du potier Dontio dans les années 65-85 (Polak, 200o). Cette fourchette de datation concorde avec les données chronologiques fournies par le reste du mobilier, à savoir la fin du ${ }_{\mathrm{r}}^{\text {er }}$ siècle, excepté le vase en céramique granuleuse $\left(\mathrm{n}^{\circ} 5\right)$ qui est attribuable à la période mérovingienne.

Outre les vases, le fossé a livré d'autres éléments remarquables : trois amas de tuiles (deux imbrices fragmentées mais complètes) et une concentration d'ossements animaux (dix restes pour $3 \mathrm{~kg}$ ). De plus, une inhumation a été mise en évidence dans la partie sud-est du fossé, suivant la même orientation.

L'hypothèse d'un fossé-limite est sous-tendue par l'analyse du parcellaire dont l'orientation perdure jusqu'à l'époque contemporaine, comme le montre la correspondance entre le fossé galloromain et une limite parcellaire figurant sur le cadastre napoléonien.

\section{Premières hypothèses}

Les sites de Soupir, Massy, Coupvray et Montmacq offrent ainsi l'exemple de six fossés ayant livré une quarantaine de vases en place [III. 13]. Il s'agit majoritairement de formes fermées de couleur sombre : pots à cuire ou vaisselle fine régionale (gobelets, bouteilles) au sein desquels on aura privilégié un gabarit standard, d'une hauteur comprise entre 13 et $21 \mathrm{~cm}$. Quelques pièces s'écartent de cette série : deux jattes et un chaudron en céramique commune sombre ainsi qu'une coupelle en sigillée. Cet assemblage est donc très éloigné de ce qu'il est habituel de trouver dans les dépotoirs d'habitat, où la diversité fonctionnelle des récipients constitue la norme.

Aucun vase n'est intact ni tout à fait complet. L'explication est soit d'ordre taphonomique, soit le résultat d'un arrachement accidentel au décapage ou d'un ramassage non exhaustif. Cependant, le cas du chaudron de Montmacq atteste que des récipients incomplets ont pu être enterrés. Par ailleurs, de nombreux pots à cuire portent systématiquement les stigmates d'une utilisation antérieure au dépôt. Il s'agit ici d'un mobilier hors d'usage qui aura été recyclé, ce dont témoigne aussi la coupelle en sigillée de Montmacq où des traces d'usure (par frottement, suite à l'empilement du vase dans un autre) ont été observées. La récupération de matériaux usagés pourrait expliquer que, paradoxalement, on ait enfoui en guise de marqueurs des objets de couleur sombre, moins facilement repérables que des cruches ou amphores dont la couleur claire aurait tranché avec celle du comblement.

Les textes antiques attestent de pratiques religieuses liées aux limites et du caractère sacré des bornes. Dans ce contexte, certaines observations peuvent prendre un tout autre sens que le simple réemploi d'objets mis au rebut. Elles invitent à établir un parallèle avec le mobilier cultuel mis au jour dans certains sanctuaires (Trescarte, 2007) : stigmates d'un passage au feu visibles sur certaines céramiques fines (non destinées à un usage culinaire ; Soupir), piquetage de la face externe d'une céramique fine (Soupir, $n^{\circ}$ 18.1), découpe de la partie supérieure d'au moins un vase (Massy), inscription gravée (Montmacq), ou encore le dépôt de vases incomplets (brisés au préalable? ; Montmacq), sans parler des fonds ou bords de vases isolés (bris rituel ?) ou bien de la présence d'un chaudron, forme rarement attestée dans les habitats. 


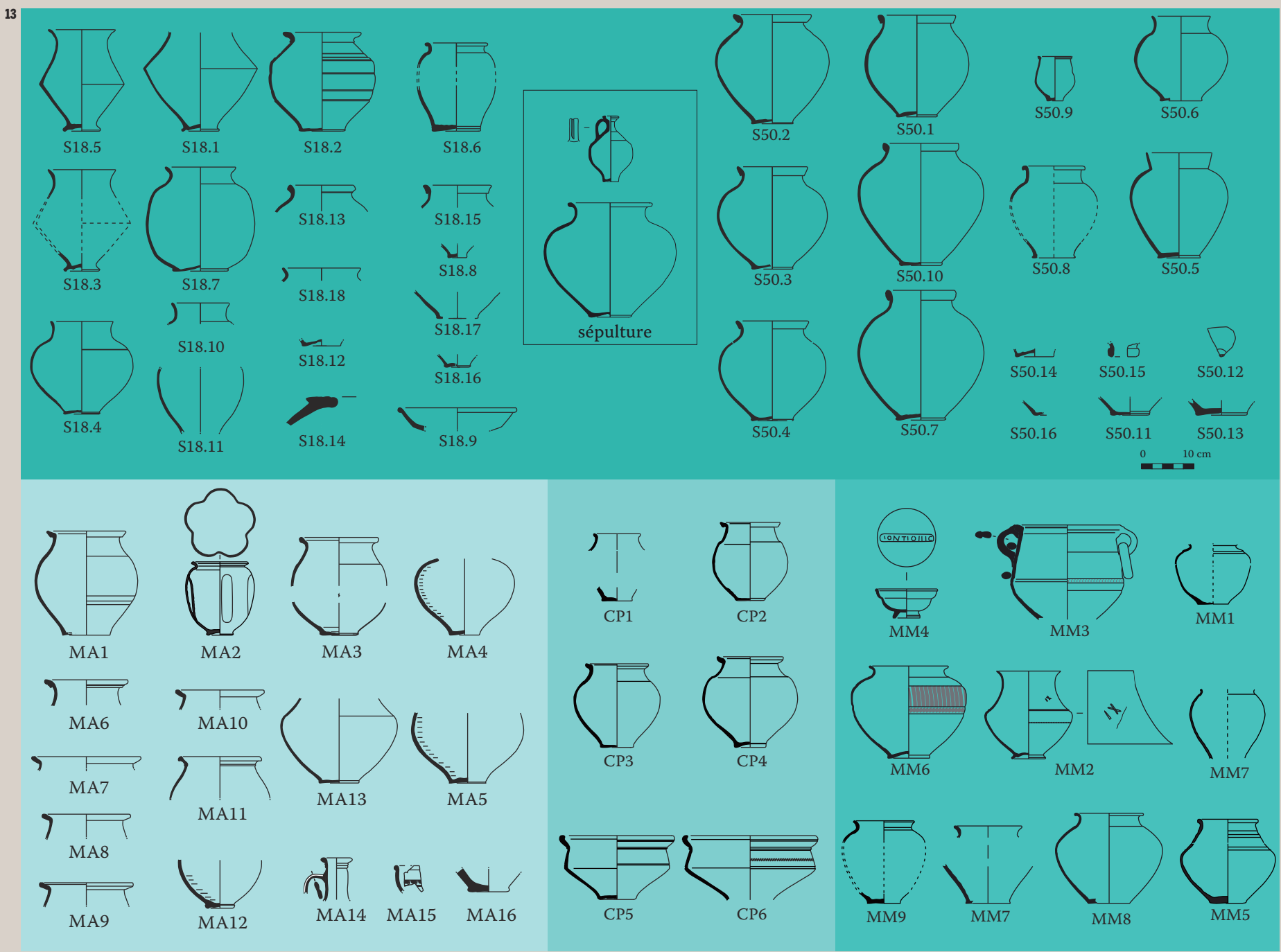

\begin{tabular}{|c|c|c|c|}
\hline & Dépôt & Fossé & Parcellaire \\
\hline Terrain & $\begin{array}{l}\text { - Mise en évidence par un suivi de fossé } \\
\text { - Repérage des éventuels dépôts } \\
\text { (éléments exogènes) } \\
\text { - Identification et enregistrement }(x, y, z) \text {, } \\
\text { photographies } \\
\text { - Vérification de la présence } \\
\text { d'un creusement préalable au dépôt } \\
\text { - Prélèvement intégral } \\
\text { (y compris de l'éventuel contenu) }\end{array}$ & $\begin{array}{l}\text { - Estimation du degré d'érosion du fossé } \\
\text { au sein de la parcelle (logs) } \\
\text { - Relevés stratigraphiques au plus près } \\
\text { du dépôt } \\
\text { - Dynamique de comblement } \\
\text { - Prélèvements micromorphologiques }\end{array}$ & $\begin{array}{l}\text { - Détermination des articulations au sein } \\
\text { de la trame parcellaire }\end{array}$ \\
\hline Postfouille & $\begin{array}{l}\text { - Remontage } \\
\text { - Dessin } \\
\text { - Photographies } \\
\text { - Caractérisation, datation, phasage } \\
\text { et organisation spatiale des dépôts } \\
\text { - Observations taphonomiques } \\
\text { - Restitution altimétrique et planimétrique } \\
\text { au sein du fossé } \\
\text { - Détermination d'aires fonctionnelles }\end{array}$ & $\begin{array}{l}\text { - Caractérisation fonctionnelle et mise } \\
\text { en évidence d'une éventuelle évolution } \\
\text { - Analyse micromorphologique }\end{array}$ & $\begin{array}{l}\text { - Rôle morphogène ou non du fossé au sein } \\
\text { du réseau parcellaire } \\
\text { - Analyse régressive du paysage } \\
\text { (résilience des formes) } \\
\text { - Intégration des foyers d'occupation } \\
\text { aux trames parcellaires antiques }\end{array}$ \\
\hline
\end{tabular}

13. Planche de synthèse

des vases de Soupir (S),

Massy (MA), Coupvray (CP)

et Montmacq (MM).

14. Protocole pour la prise

en compte des dépôts

sur le terrain et en phase

postfouille. 
Quelle place pourraient occuper aussi dans ce dispositif de bornage les sépultures mises au jour dans ou à proximité des fossés de Soupir et Montmacq, sachant que les textes nous renseignent sur leur utilisation comme bornes ainsi que sur leur inviolabilité, qui leur confère un rôle de protection des limites ? Les mêmes questions se posent à propos des tessons isolés, des restes fauniques, amas de tuiles ou des pierres dont la présence, sur les sites de Massy et Montmacq, ne paraît pas aléatoire : une plus grande densité a été notée là où précisément les vases entiers sont plus rares.

Enfin, des raisons d'ordre purement fonctionnel, liées à l'entretien des fossés, ont pu aussi présider à l'enfouissement des vases (Massy).

Les occurrences analysées sont encore trop peu nombreuses pour valider ces hypothèses. Des progrès significatifs dans la compréhension de ces dépôts au sein de fossés ne sont envisageables quà la condition de constituer un corpus d'étude plus large (une quarantaine d'autres cas ont d'ores et déjà été recensés). Cela passe aussi par l'élaboration d'un protocole d'analyse à trois échelles d'observation distinctes (le dépôt luimême, le dépôt au sein du fossé et la place occupée par ce dernier au sein du parcellaire) [ill. 14] ainsi que par la prise en compte d'un éventuel marqueur de surface, tel celui de Saint-Martin-deBréthencourt, « Les Terres Salées » (Brutus, 2013), où la borne a été conservée en place avec son dépôt céramique sous-jacent.

\section{Perspectives}

On a pu observer des cas de figure variés où le fossé, excepté dans un cas, est relativement éloigné de tout habitat connu (300 m pour le plus proche) et semble matérialiser soit une limite entre propriétés foncières (Soupir, fossé sud de Coupvray), soit une limite d'emprise publique d'une voie de grand parcours (Massy), soit la limite interne d'une propriété (entre secteurs d'habitation et d'activité ; fossé nord de Coupvray), soit encore la limite forte d'un site antique encore mal défini (Montmacq). Les dépôts participeraient ainsi à la volonté de pérenniser un tracé important que l'abandon des fossés menace de disparition, d'effacement.

Si l'on a mis en évidence la présence de vases en dépôt au niveau d'apparition de fossés-limites et la fréquence d'un certain type de vase (forme fermée de couleur sombre et de taille moyenne), faut-il pour autant envisager que des gestes très normalisés aient présidé à l'opération de bornage à l'intérieur d'un vaste territoire tel que celui étudié ici, qui englobe le sud de la Picardie et l'Île-deFrance ? Ces dépôts renvoient-ils à des pratiques isolées, voire anecdotiques, ou bien leur importance est-elle sous-estimée ? Et qu'en est-il des autres repères (moellons de pierre, fragments de tuiles, ossements animaux, sépultures...) ainsi que de la cérémonie entourant ces opérations de délimitation? Autant de questions qui pourront être éclairées par la mise en œuvre d'un protocole spécifique...

\section{Références bibliographiques}

Bruant J., Brunet P., Cammas C., Griselin S MouChÊne-Boris Ch., 2008: Massy-Rue du Pérou, Rapport de diagnostic archéologique, Inrap/SRA Île-de-France, Saint-Denis.

Bruant J., 2009: «Étude d'un tronçon de l'axe ParisOrléans », Archéopages. Voies et Réseaux, ${ }^{\circ}$ 27, p. 58-59. Bruant J., Cotté O., Desrayaud G., Pissot V., TOUQUET R, 2011: «Vases entiers dans des fossés : des pratiques liées au bornage des terres? Présentation de trois exemples franciliens », Actes $d u$ Congrès de la SFECAG, Arles, 2-5 juin 2011, Marseille, SFECAG, p. 585-595.

Brutus F., 2013 : Saint-Martin-de-Bréthencourt. Les Terres Salées (Yvelines), Rapport de diagnostic archéologique, Inrap/SRA Île-de-France.

Coulon G., 2007: Les voies romaines en Gaule, Paris, éd. Errance (coll. Promenades archéologiques).

DERU X., 1996 : La céramique belge dans le nord de la Gaule. Caractérisation, chronologie, phénomènes culturels et économiques, Louvain-la-Neuve, Université catholique de Louvain, Département d'archéologie et d'histoire de l'art, 463 p.

DurAND S., 2012 : Coupvray, Montry, Magny-le-Hongre, ZAC des Trois Ormes, Rapport de fouilles, Inrap/ SRA Île-de-France, 2 vol.
Ellis J.B., Chocat B., Fujita S., Marsalek J., RAUCH W. (DIR.), 2004: Urban Drainage. A Multilingual Glossary: English, French, German, Japanese, Londres, IWA Publishing, $528 \mathrm{p}$.

Favory F., Gonzales A., Guillaumin J.-Y., Robin P., 1995 : « Témoignages antiques sur le bornage romain II », RACF, 34, p. 261-281.

Gurrera M., Gallemí F., 1994 : « El jaciment de Can Soleret (Mataró, Maresme). Un límit de proprietat rural privada en época romana al Maresme », Laietania, 9, p. 159-18o.

Hénon B., Boulen M., Flucher G., Robert B., 2001 : "Soupir "Petite Forêt" (o2), fouilles 2001 », Sauvetage archéologique dans la vallée de l'Aisne 2001 Document final de synthèse, Inrap/SRA Picardie.

MAUNÉ S., 1992 : « Le site de Sept-Fonts (Saint-Pons-deMauchiens, Hérault). Une limite de propriété augustéenne? ?, Dialogues d'histoire ancienne (DHA), 18, 2, p. 313-317.

MAUNÉ S., 1997 : « Un lot de céramique d'époque augustéenne à Sept-Fonts (Saint-Pons-deMauchiens, Hérault) », Actes du Congrès de la SFECAG, Le Mans, 8-11 mai 1997, Marseille, SFECAG, p. $457-480$.

Olesti V. O., Massó J., 1997 : « Une limite de propriété rurale dans l'ager Tarraconensis », $D H A, 23-2$, p. 224-232.
PARIS C., 2012 : Montmacq, Oise, rue de la Croix Noire, Rapport de diagnostic, Inrap Nord-Picardie.

POLAK M., 2000 : South Gaulish Terra Sigillata with Potter's Stamps from Vechten, Nijmegen, Katholieke Universiteit Nijmegen (coll. Rei Cretariae Romanae Fautorum Acta. Supplementum, 9).

ROBERT S., 1996: « Le parcellaire du plateau de Sénart (Seine-et-Marne). Les formes du paysage. Tome 1 », in Chouquer G. (DIR.), Études sur les parcellaires, Paris, éd. Errance, p. 11-26.

Robert B. (en cours) : Moussy-Verneuil (Aisne), Rapport de diagnostic 2012, Inrap Nord-Picardie.

TrescarTe J., 2007: « Céramiques communes en contexte cultuel : le cas du Puy-de-Dôme, sanctuaire sommital et agglomération du col de Ceyssat (Puyde-Dôme) », Actes du Congrès de la SFECAG, Langres, 17-20 mai 2007, Marseille, SFECAG, p. 367-394

Vidal L., Рetitot H., 2003 : « Pour une archéologie de la limite et du bornage. Données antiques de la Gaule Narbonnaise », Actualité de la Recherche en Histoire et Archéologie agraires. Actes du colloque international AGER V de Besançon, 18-22 septembre 200o, Besançon, Presses universitaires franc-comtoises (coll. Annales littéraires, 764 ; Série «Environnement, sociétés et archéologie », 5), p. 79-96. 